\title{
Higgs shifts from electron-positron annihilations near neutron stars
}

\author{
Gary A. Wegner ${ }^{1, a}$, Roberto Onofrio ${ }^{2,3, b}$ \\ ${ }^{1}$ Department of Physics and Astronomy, Dartmouth College, 6127 Wilder Laboratory, Hanover, NH 03755, USA \\ 2 Dipartimento di Fisica e Astronomia 'Galileo Galilei', Università di Padova, Via Marzolo 8, 35131 Padova, Italy \\ ${ }^{3}$ ITAMP, Harvard-Smithsonian Center for Astrophysics, 60 Garden Street, Cambridge, MA 02138, USA
}

Received: 6 March 2015 / Accepted: 14 June 2015 / Published online: 4 July 2015

(C) The Author(s) 2015. This article is published with open access at Springerlink.com

\begin{abstract}
We discuss the potential for using neutron stars to determine bounds on the Higgs-Kretschmann coupling by looking at peculiar shifts in gamma-ray spectroscopic features. In particular, we reanalyze multiple lines observed in GRB781119 detected by two gamma-ray spectrometers, and derive an upper bound on the Higgs-Kretschmann coupling that is much more constraining than the one recently obtained from white dwarfs. This calls for targeted analyses of spectra of gamma-ray bursts from more recent observatories, dedicated searches for differential shifts on electron-positron and proton-antiproton annihilation spectra in proximity of compact sources, and signals of electron and proton cyclotron lines from the same neutron star.
\end{abstract}

\section{Introduction}

The interplay between the mass generation through the Higgs field and gravitation is an active subfield of investigation and the concept of mass is crucial both in quantum field theory and gravitation. Consequently, any common insight may shed light on the possible unification of the two theories. Inflation, although successful in removing potential contradictions to the standard Big Bang model, does not yet have a clear microscopic interpretation and unambiguous observational evidence, and the Higgs field has been conjectured to be responsible for it $[1,2]$. To these long standing motivations, others have been added since the discovery at the LHC of a scalar particle with the mass and decay branching ratios as expected from the Higgs boson in the minimal Higgs doublet model $[3,4]$. In the absence of signals for new physics in the current experimental setting, the extrapolation of the standard model to the Planck scale raises an issue of stability of the vacuum for the specific value of the quartic coupling of the Higgs self-interaction which is extracted

\footnotetext{
a e-mail: gary.a.wegner@dartmouth.edu

b e-mail: onofrior@gmail.com
}

from its observed mass [5-8]. Various recent contributions point out a solution with no new physics beyond the standard model apart from a non-minimal coupling of the Higgs field to curvature invariants [9-11]. Finally, the tentative interpretation of the BICEP-2 results [12] in terms of gravitational waves produced during inflation, still to be fully scrutinized and compared with Planck results [13], would call for nonminimal coupling of the Higgs field to gravity [14] or to the inflaton [15].

Recently, we discussed upper bounds to the coupling between the Higgs field and a specific curvature invariant, the Kretschmann invariant, based on the analysis of molecular lines of $C_{2}$ and atomic lines of $\mathrm{H}, \mathrm{C}, \mathrm{Ca}$, and $\mathrm{Mg}$ from the surface of two white dwarfs [16]. This bound, although already competitive with respect to what is achievable in table-top experiments, in principle may be improved by many orders of magnitude by exploiting the strong gravity at the surfaces of neutron stars. The boost in sensitivity is easily estimated as the Kretschmann invariant $K=R_{\mu \nu \rho \sigma} R^{\mu \nu \rho \sigma}$, with $R^{\mu \nu \rho \sigma}$ the Riemann curvature tensor, depends on the sixth power of the radius of the astrophysical object. Thus, for an Earth-radius white dwarf and a $10 \mathrm{~km}$ neutron star of equal mass, a gain of order $(6000 / 10)^{6} \simeq 4.7 \times 10^{16}$ is expected if spectral features could be measured with precision comparable to the ones studied in white dwarfs. The crucial issue is to get measurable features from neutron star spectra, and in the following we discuss possibilities to be considered, in the form of reanalysis of already collected data, and future dedicated observations. In Sect. 2 we consider annihilation lines tentatively observed during gammaray bursts with redshifts compatible to that expected from the surface of neutron stars, and discuss the possibility for an anomalous contribution with respect to the redshifts due to lines of nuclear origin. In Sect. 3 this evidence is conservatively considered as a bound on a possible signal due to the Higgs shift, and the related bounds discussed. In Sect. 4 we discuss possible Higgs shifts arising from a comparative 
analysis of electron-positron and proton-antiproton annihilations, always in proximity of a neutron star, which should result in similar bounds to the one discussed earlier. More general considerations on the possibility of observing simultaneously cyclotron lines from electrons and protons are discussed in the conclusions, as well as possible generalizations of the Higgs coupling in theories and models beyond general relativity.

\section{Search for anomalous shifts in gamma-ray bursts}

The most likely avenue toward obtaining better bounds to Higgs-Kretschmann couplings from signals in proximity of neutron stars is, to our knowledge, the comparative analysis of electron-positron annihilation lines and narrow lines due to nuclear de-excitations during transients of gammaray bursts. In the following we focus on the event collected by the Goddard germanium Gamma-Ray Burst Spectrometer on board of ISEE-3, GRB 781119, [17,18], as several lines attributed to nuclei like ${ }^{56} \mathrm{Fe},{ }^{24} \mathrm{Mg},{ }^{20} \mathrm{Ne},{ }^{28} \mathrm{Si},{ }^{14} \mathrm{~N}$ were identified. A less prominent peak at $420 \mathrm{keV}$ has also been identified and interpreted as a redshifted $e^{+} e^{-}$annihilation peak. The same event was observed by the Konus gamma-ray observatory of the Leningrad group at the Ioffe Institute $[19,20]$, including the emission feature at $420 \mathrm{keV}$. The redshift required to justify this line as a $e^{+} e^{-}$annihilation peak is compatible with the gravitational redshift $(z \simeq 0.2)$ expected on the surface of a neutron star. More qualitatively, the putative electron-positron line is observed at $E_{\text {annih }}=(420 \pm 20) \mathrm{keV}$, which is $0.82 \pm 0.04$ times the value of the electron-positron two-body annihilation line at $511 \mathrm{keV}$. For the ${ }^{56} \mathrm{Fe}$ line, we have an observed value of $E_{\mathrm{Fe}}=(738 \pm 10) \mathrm{keV}$ versus an unshifted energy of $847 \mathrm{keV}$, i.e. an energy ratio of $0.87 \pm 0.01$. Therefore the electron-positron line is more redshifted than the ${ }^{56} \mathrm{Fe}$ line by one standard deviation. Although the discrepancy between the two redshifts is contained within one standard deviation only, by inferring the redshifts of the other identified nuclear lines from Fig. 6 in [18] we notice that they all result in systematically smaller redshifts than the electron-positron annihilation line, at the level of 4.2 standard deviations, as discussed in Table 1.

While it is tempting to interpret this extra shift as due to the Higgs shift, more conservatively it can be used to determine an upper bound on the associated Higgs-Kretschmann coupling, for two reasons.

The interpretation of the $420 \mathrm{keV}$ peak as due to electronpositron annihilation is not solid, and needs to be corroborated by more data. There is a general consensus that spectral features in the $300-400 \mathrm{keV}$ region can be interpreted as due to a gravitationally redshifted $e^{+} e^{-}$annihilation line; see [22] for observations of the galactic center and [23] for their
Table 1 Analysis of the line shifts from the ISEE-3 data for the gammaray burst event recorded on 19 November 1978 reported in [18]. The origin of each line is in the first column, followed by the transition energy as measured in the laboratory [21], the one observed by ISEE-3, and the related redshift evaluated as $z_{\text {line }}=E_{\mathrm{lab}} / E_{\mathrm{obs}}-1$. The error bars are evaluated from our analysis of full-width half maxima of the interpolating curves appearing in Fig. 6 of [18], and they are used to obtain weighted average and standard deviation on the eight redshift determinations for the nuclear lines, resulting in $\left\langle z_{\text {nucl }}\right\rangle=0.134 \pm 0.017$, which is smaller by 4.2 standard deviations with respect to the one evaluated using the electron-positron annihilation peak, $z_{\text {annih }}=0.217 \pm 0.010$. The instrumental error for the nuclear transitions reported in [18] is quoted only for the ${ }^{56} \mathrm{Fe}$ line as $10 \mathrm{keV}$, corresponding to a relative error of $\simeq 1.3 \%$. If we assume the same instrumental error for all remaining nuclear lines, then this last is smaller by one order of magnitude with respect to the statistical relative error on $\left\langle z_{\text {nucl }}\right\rangle$, and we get $\left\langle z_{\text {nucl }}\right\rangle=0.143 \pm 0.018$, which differs by 3.6 standard deviation from $z_{\text {annih }}$, corroborating the former analysis based on our graphical assessment of the error bars in Fig. 6 of [18]

\begin{tabular}{lcrl}
\hline Line & $E_{\text {lab }}(\mathrm{keV})$ & \multicolumn{1}{c}{$E_{\text {obs }}(\mathrm{keV})$} & \multicolumn{1}{l}{ line } \\
\hline$e^{+} e^{-}$ & 511 & $420 \pm 20$ & $0.217 \pm 0.010$ \\
${ }^{56} \mathrm{Fe}$ & 847 & $738 \pm 40$ & $0.148 \pm 0.008$ \\
${ }^{56} \mathrm{Fe}$ & 1238 & $1076 \pm 33$ & $0.151 \pm 0.005$ \\
${ }^{24} \mathrm{Mg}$ & 1369 & $1164 \pm 36$ & $0.176 \pm 0.005$ \\
${ }^{20} \mathrm{Ne}$ & 1634 & $1444 \pm 33$ & $0.132 \pm 0.003$ \\
${ }^{28} \mathrm{Si}$ & 1779 & $1589 \pm 33$ & $0.120 \pm 0.002$ \\
${ }^{56} \mathrm{Fe}$ & 1811 & $1612 \pm 40$ & $0.123 \pm 0.003$ \\
${ }^{14} \mathrm{~N}$ & 2313 & $2011 \pm 70$ & $0.150 \pm 0.005$ \\
\hline
\end{tabular}

interpretation. However, the absence of similar signals in later observational campaigns like BATSE strongly constrains the initial interpretation of the observed peaks [24-26]. On the instrumental side, the energy deposition of each photon may not be completely occurring within the detector, and therefore assumptions must be made on the incident spectrum [27-29]. The very existence of the line features has been criticized [30,31], and alternative explanations have been put forward, more specifically as originating from de-excitation of ${ }^{7} \mathrm{Li}^{*}$ in cosmic rays [32] and from an amplification mechanism through stimulated annihilation radiation [33].

Moreover, various environmental factors might create differential shifts between the $e^{+} e^{-}$annihilations and the nuclear lines as there are uncertainties in the models of gamma-ray bursts in neutron stars. Although it is reasonable to assume that the nuclear lines originate from matter on the surface of the neutron star with null Doppler shift, peculiar motions of the $e^{+} e^{-}$plasma clouds may add or subtract a Doppler shift to the gravitational redshift. The broadening and shift due to the finite temperature of the $e^{+} e^{-}$plasma cloud could reduce the observed redshift. The annihilation line broadens proportionally to $T^{1 / 2}$ for $k_{\mathrm{B}} T<<m_{e} c^{2}$, and to $T$ for $k_{\mathrm{B}} T>>m_{e} c^{2}$, and the peak of the line shifts toward higher energies as $\delta E_{\text {peak }} / E_{\text {peak }} \simeq 1.25 k_{\mathrm{B}} T / m_{e} c^{2}$ [34-38]. This blueshift is going to aggravate the redshift excess we 
Table 2 Analysis of the line shifts from emission features in the 70$470 \mathrm{keV}$ interval from the Konus catalogs [39-41]. The date of the event for each line is in the first column, followed by the observed emission energy, the corresponding redshift if attributed to the $e^{+} e^{-}$annihilation peak, and the $M / R$ parameter expressed in units of $c^{2} /\left(2 G_{N}\right)$

\begin{tabular}{llll}
\hline Event & $E_{\text {obs }}(\mathrm{keV})$ & $z_{\text {line }}$ & $\mathrm{M} / \mathrm{R}$ \\
\hline $18 / 09 / 78$ & 380 & 0.345 & 0.447 \\
$21 / 09 / 78$ & 350 & 0.460 & 0.531 \\
$06 / 10 / 78 \mathrm{a}$ & 420 & 0.217 & 0.324 \\
$06 / 10 / 78 \mathrm{~b}$ & 350 & 0.460 & 0.531 \\
$23 / 10 / 78$ & 280 & 0.825 & 0.700 \\
$05 / 03 / 79$ & 380 & 0.345 & 0.447 \\
$06 / 04 / 79$ & 320 & 0.597 & 0.608 \\
$02 / 05 / 79$ & 470 & 0.087 & 0.154 \\
$26 / 05 / 79$ & 320 & 0.597 & 0.608 \\
$22 / 06 / 79$ & 450 & 0.136 & 0.224 \\
$28 / 06 / 79$ & 410 & 0.246 & 0.356 \\
$09 / 11 / 79$ & 320 & 0.597 & 0.608 \\
\hline
\end{tabular}

have discussed. It is also worth remarking that a secondary peak on the right side of the putative $e^{+} e^{-}$annihilation peak is present at an energy of $484 \mathrm{keV}$ in the ISSE-3 data [18]. If this is interpreted as the actual $e^{+} e^{-}$annihilation peak, the corresponding redshift is only $z_{\text {annih }}=(0.056 \pm 0.002)$, blueshifted by the average value of the redshift from nuclear lines by about 4.3 standard deviations. Based on the redshift of the nuclear lines, a temperature of the $e^{+} e^{-}$plasma cloud of $\simeq 20 \mathrm{keV}$ (corresponding to $\simeq 2.6 \times 10^{8} \mathrm{~K}$ ) should be required to fully justify this blueshift, which seems compatible with the typical surface temperature of neutron stars.

A statistical comparison between the distribution of the energy of the putative $e^{+} e^{-}$annihilation peaks in gamma-ray bursts and the mass-radius distribution of neutron stars may also be used both to check the hypotheses attributing these events to neutron stars and to check for systematic deviations. The mass-radius ratio is related to the energy of the observed line and the redshift as$$
\frac{M}{R}=\frac{c^{2}}{2 G_{N}}\left[1-\left(\frac{E_{\mathrm{obs}}}{E_{\mathrm{lab}}}\right)^{2}\right]=\frac{c^{2}}{2 G_{N}}\left[1-\frac{1}{(1+z)^{2}}\right] .
$$

The Konus collaboration has published three catalogs of gamma-ray bursts [39-41], and in 25 cases we find evidence for emission peaks at energies in the $100-470 \mathrm{keV}$ range. Within this subsample of events, we apply a lower model-dependent cutoff based on the requirement for causality $[42,43]$. If the events originate on the surface of a neutron star, this requires $M / R \leq 0.708 c^{2} /\left(2 G_{N}\right)$ leading to $E_{\text {obs }} \geq 276 \mathrm{keV}$. The resulting events are reported in Table 2. This leads to a statistical distribution of the anni-

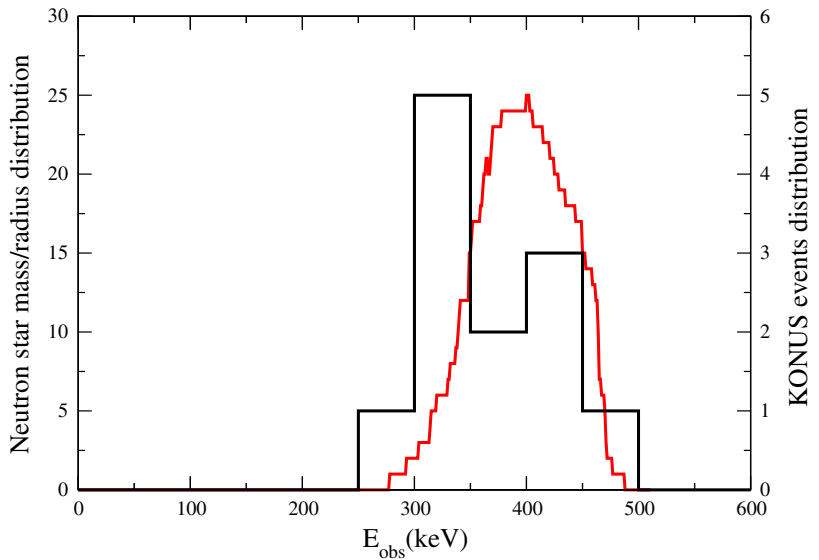

Fig. 1 Statistics of the distribution of the observed energy $E_{\mathrm{obs}}$. Distribution of the redshifts ratio as deduced from the emission lines of the Konus catalog, see Table 2, in the hypotheses they represent $e^{+} e^{-}$ annihilation lines (black line, right vertical scale), and from 26 determinations of gravitational redshifts for neutron stars [44-46] (red line, left vertical scale)

hilation energy peaks, depicted in Fig. 1, which is also compared with the mass-to-radius ratio distribution for a sample of neutron stars obtained from [44-46], considering a range of values for $E_{\text {obs }}$ coming from the allowed intervals for mass and radius (see [44-46] for a discussion of the errors in the determination of mass and radius, and their sensitivity to the equations of state) and summing up the resulting rectangular windows of unit height. The two distributions are at least limited by roughly the same interval, but the Konus distribution seems more peaked at lower energies. The small number of events in the Konus data prevents us to make more quantitative analyses. ${ }^{1}$

\section{Bounds on the Higgs-Kretschmann coupling}

As detailed in former contributions $[16,47,48]$, if the Higgs field $\phi$ characterized by quadratic and quartic coefficients $\mu$ and $\lambda$ is coupled to the Kretschmann curvature invariant $K$ via the Lagrangian density term $\xi_{K} \Lambda_{\mathrm{Pl}}^{2} \phi^{2} K$, with $\xi_{K}$ their coupling constant and $\Lambda_{\mathrm{Pl}}$ the Planck length, the effective mass parameter of the Higgs field gets an extra-term due to the scalar curvature as $\mu^{2} \mapsto \mu^{2}\left(1+\xi_{K} \Lambda_{\mathrm{Pl}}^{2} \lambda_{\mu}^{2} K\right)$, with $\lambda_{\mu}$ the Compton wavelength of the Higgs field corresponding to its mass of $125 \mathrm{GeV}\left(\lambda_{\mu}=1.6 \times 10^{-18} \mathrm{~m}\right)$, and the vacuum expectation value of the Higgs field $v$ depends on space as

\footnotetext{
${ }^{1}$ We have analyzed the low energy spectra of the Konus-Wind data for the year 2003 publicly available at the NASA website: http://asd. gsfc.nasa.gov/konus/, with no evidence for spectral features apart from a recurrent peak at about $260 \mathrm{keV}$ with approximately constant height, presumably of instrumental origin.
} 
$v=\sqrt{-\frac{\mu^{2}+\xi_{K} \Lambda_{\mathrm{Pl}}^{2} K}{\lambda}} \simeq v_{0}\left(1+\frac{\xi_{K} \Lambda_{\mathrm{Pl}}^{2} K}{2 \mu^{2}}\right)$,

in a weak-curvature limit, i.e. with curvature length scale much larger than $\lambda_{\mu}$, a limit well satisfied in all circumstances of astrophysical interest. Then the mass $m_{e}$ of the electron will be simply changed proportionally to the Higgs vacuum expectation value

$\delta m_{e}=\frac{y_{e}}{\sqrt{2}}\left(v-v_{0}\right) \simeq \frac{y_{e} \xi_{K} \Lambda_{\mathrm{Pl}}^{2} K v_{0}}{2^{3 / 2} \mu^{2}}=\frac{1}{2} \xi_{K} \Lambda_{\mathrm{Pl}}^{2} \lambda_{\mu}^{2} K m_{e}$.

where $y_{e}$ is the Yukawa coupling of the electron. Hadrons, having most of their mass arising from QCD vacuum, are instead minimally affected by the curvature invariant. In order to get upper bounds based on the data above, we first check that the Higgs shift expected for the nuclear lines is indeed negligible with respect to the one expected in the $e^{+} e^{-}$ annihilation peak. The relationship between the mass of a nucleon and the energy levels of the corresponding nucleus is not trivially available, as there are many phenomenological models based either on a single nucleon approach, in which a nucleon evolves in the mean-field potential created by the remaining nucleons, as in the shell model approach, or on a many-body collective approach as in the droplet model [49]. Considering that strong interactions are mainly responsible for the binding between nucleons, we assume that in a simplified treatment with a harmonic oscillator potential, the energy levels will scale as the inverse of the square root of the involved mass, the mass of the nucleon in the shell models, and the whole mass of the nucleus in collective models. In this case the expected Higgs shift should scale as $\delta \lambda / \lambda \simeq \delta m_{n} /\left(2 m_{n}\right)$, with $m_{n}$ the relevant mass (ranging between the two extreme values of the nucleon mass, for instance the one of the proton $m_{p}$, and the nucleus mass $m_{N}$ ). In an infinite square-well model instead the expected scaling should be the inverse of the mass, $\delta \lambda / \lambda \simeq \delta m_{n} / m_{n}$, a mere factor 2 larger than in the harmonic potential.

In the case of a single nucleon, relevant for single-particle models such as the shell model, we have, focusing on the proton mass, $m_{p} \simeq m_{\mathrm{QCD}}+\left(2 y_{u}+y_{d}\right) v / \sqrt{2} \simeq 928+$ $10\left(1+0.5 \xi_{K} \Lambda_{P}^{2} \lambda_{\mu}^{2} K\right)$ (with $y_{u}$ and $y_{d}$ the Yukawa couplings of the up and down quarks, $m_{\mathrm{QCD}}$ the purely gluonic contribution to the proton mass, and all masses and energies expressed in $\mathrm{MeV} / \mathrm{c}^{2}$ ), which implies $\delta m_{p} / m_{p} \simeq$ $5 \times 10^{-3} \xi_{K} \Lambda_{P}^{2} \lambda_{\mu}^{2} K$. This has to to be compared with the sensitivity to the Higgs-Kretschmann coupling of the electron, $\delta m_{e} / m_{e} \simeq 0.5 \xi_{K} \Lambda_{P}^{2} \lambda_{\mu}^{2} K \simeq 10^{2} \delta m_{p} / m_{p}$. In order to estimate the mass shift in the case of collective models, we consider the nucleus with the larger number of lines observed as in Table 1, the iron isotope with mass number 56 made of 26 protons and 30 neutrons. Its mass can be written in terms of the Yukawa couplings $y_{u}$ and $y_{d}$ of the up and down valence quarks inside protons and neutrons obtaining

$m_{\mathrm{Fe}}=56 m_{\mathrm{QCD}}+\frac{\left(82 y_{u}+86 y_{d}\right) v}{\sqrt{2}}-\Delta m(A, Z)$,

where we have introduced the mass defect $\Delta m(A, Z)$. In the presence of a curved spacetime with Kretschmann coupling to the Higgs field the mass of a ${ }^{56} \mathrm{Fe}$ nucleus will be

$m_{\mathrm{Fe}}\left(\xi_{K}\right)=m_{\mathrm{Fe}}(0)\left(1+\frac{82 y_{u}+86 y_{d}}{2 \sqrt{2}} \xi_{K} \Lambda_{P}^{2} \lambda_{\mu}^{2} K\right)$.

Again, the relative mass shift of the nucleus turns out to be smaller than the relative mass shift of the electron by a factor even larger than in the single nucleon case, since $\left(\delta m_{e} / m_{e}\right) /\left(\delta m_{\mathrm{Fe}} / m_{\mathrm{Fe}}\right) \simeq \sqrt{2} m_{\mathrm{Fe}} /\left(82 y_{u}+86 y_{d}\right) v_{0} \simeq 87$. Therefore, both the extreme examples of the single nucleon mass and the whole ${ }^{56} \mathrm{Fe}$ mass determining the nuclear spectroscopy show that their contributions are negligible with respect to the mass shift of the electron, the nuclear line thus providing a spectroscopic 'anchor'. This implies that we may attribute the Higgs shift to the electron mass shift alone and, by assuming the Planck length $\Lambda_{P}=10^{-35}$ $\mathrm{m}$ and a solar mass neutron star with radius $10 \mathrm{~km}$ (corresponding to a Kretschmann invariant on the neutron star surface of $K=10^{-16} \mathrm{~m}^{-4}$ ), the maximum Higgs shift compatible with the observed excess of redshift $\delta z=z_{\text {annih }}-$ $\left\langle z_{\text {nucl }}\right\rangle \geq 1.28 \times 10^{-122} \xi_{K}$, gives an upper bound on the Higgs-Kretschmann coupling coupling $\xi_{K} \leq 5.8 \times 10^{120}$ in MKSA units, translated into a value of $7.1 \times 10^{35}$ in natural units. If the line at $484 \mathrm{keV}$ is instead considered responsible for the $e^{+} e^{-}$annihilation, the Higgs shift is negative, $\delta z=z_{\text {annih }}-\left\langle z_{\text {nucl }}\right\rangle=-0.087 \pm 0.018$, corresponding to an upper bound of $\xi_{K} \geq-6.8 \times 10^{120}$ (i.e. $\left|\xi_{K}\right| \leq 6.8 \times 10^{120}$ ), always in MKSA units. By assuming $\Lambda_{P}=10^{-19} \mathrm{~m}$ as in models with the Planck scale coinciding with the Fermi scale [50,51], the bounds are correspondingly stronger by a factor $\simeq 10^{32}$.

\section{Electron-positron and proton-antiproton annihilations near neutron stars}

An alternative possibility to study Higgs shifts is to compare electron-positron and proton-antiproton annihilation signals from neutron stars, as suggested in [47]. Unlike $e^{+} e^{-}$annihilations, $p \bar{p}$ annihilations produce a continuous photon spectrum since the annihilation produces multiple $\pi^{0}$ mesons in turn decaying into photons. Consequently the photon spectrum has an intrinsically broad peak due to the more than 
two-body decays, which is further Doppler-broadened by the velocity spread of the involved particles. Due to the flatness of the expected photon spectrum from $p \bar{p}$ annihilation, possible limits on the Kretschmann coupling from this class of events are weaker than the former class of events. The continuous gamma-ray spectrum in the $p \bar{p}$ annihilations has been evaluated in [52] by fitting a Monte Carlo simulation with an analytical function as

$F\left(E_{\gamma}\right)=N\left[\left(E_{p}-E_{\gamma}\right)^{\alpha_{1}} e^{\beta_{1}}+\left(E_{p}-E_{\gamma}\right)^{\alpha_{2}} e^{\beta_{2}}+\beta_{3} e^{\alpha_{3} E_{\gamma}}\right]$,

where $E_{p}=m_{p} c^{2}$ is the proton mass in energy units, and $\alpha_{i}, \beta_{i}(i=1 \div 3)$ are fitting parameters available in [52]. The position of the annihilation peak depends upon the assumed proton mass, as shown in Fig. 2, with a best fit yielding $\bar{E}_{\gamma}=187.05-0.12385 E_{p}$ (all energies in $\mathrm{MeV}$ ), with the slope $\delta \bar{E}_{\gamma} / \delta m_{p}=0.12385$ expressing its sensitivity. If the minimum detectable peak shift is then $\delta \bar{E}_{\gamma} \simeq 0.1$ $\mathrm{MeV}$, the minimum detectable proton mass shift is $\simeq 0.8$ $\mathrm{MeV}$, i.e. $\delta m_{p} / m_{p} \simeq 8 \times 10^{-4}$, and the situation is similar to the one in the previous section as the sensitivity of the proton shift to the spacetime curvature is far smaller than the one of the electron. For the electron-positron annihilations, the limitation is due to the intrinsic resolution of the $511 \mathrm{keV}$ peak which depends upon the environmental temperature and energy resolution of the detector, estimated, respectively, to be $\Delta E /\left.E\right|_{\text {env }}=K_{\mathrm{B}} T / E_{\gamma} \simeq 10^{-5}$, and $\Delta E /\left.E\right|_{\text {instr }}=1.47 \times 10^{-4}[53]$. By using the Rayleigh criterion for resolving a shift of the annihilation peak, with a full-width half maximum of $2.37 \mathrm{keV}$ as quoted in [53], and the same values of $\Lambda_{\mathrm{Pl}}, \lambda_{\mu}$, mass and radius of the neutron star used above, we get a bound $\xi_{K}=4.4 \times 10^{34}$ using natural

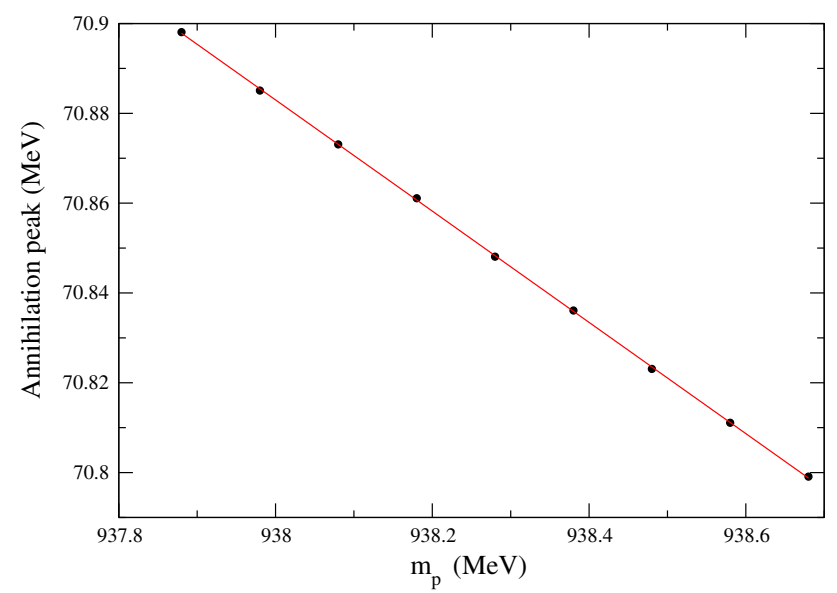

Fig. 2 Sensitivity of the energy distribution peak in $p \bar{p}$ annihilations to the proton mass. The fit yields $\bar{E}_{\gamma}=187.05-0.12385 m_{p}$, with energies and the proton mass expressed in $\mathrm{MeV}$
Table 3 Summary of bounds on the Higgs-Kretschmann couplings (in natural units) from various sources, experiments to test the superposition principle of the gravitational force as discussed in [48], the analysis of differential shifts in spectral lines from two white dwarfs [16], the analysis of differential shifts between $e^{+} e^{-}$annihilation line and nuclear lines with two candidates at $420 \mathrm{keV}$ (a) and $484 \mathrm{keV}$ (b) as discussed in Sect. 3, and possible comparisons between $e^{+} e^{-}$and $p \bar{p}$ annihilation lines originating from the surface of neutron stars, as discussed in Sect. 4. The bounds are evaluated for a choice of the Planck length of $\Lambda_{\mathrm{Pl}}=10^{-35} \mathrm{~m}$, the bounds for the choice of $\Lambda_{\mathrm{Pl}}=10^{-19} \mathrm{~m}$ being $10^{32}$ times stronger

\begin{tabular}{lc}
\hline Source & $\xi_{K}\left(\Lambda_{\mathrm{Pl}}=10^{-35} \mathrm{~m}\right)$ \\
\hline Table-top experiments & $2.5 \times 10^{60}$ \\
BPM 27606 & $5 \times 10^{50}$ \\
Procyon B & $9 \times 10^{50}$ \\
$e^{+} e^{-}$vs. nuclei (a) & $7.1 \times 10^{35}$ \\
$e^{+} e^{-}$vs. nuclei (b) & $-8.4 \times 10^{35}$ \\
$e^{+} e^{-}$vs. $p \bar{p}$ & $4.4 \times 10^{34}$ \\
\hline
\end{tabular}

units, one order of magnitude stronger than to the one evaluated above for the gamma-ray burst event. Table 3 summarizes our discussion by including upper bounds from laboratory measurements, actual spectroscopy from white dwarfs, and the potential observations from neutron stars reported in this paper.

\section{Conclusions}

In summary, we have discussed neutron stars as potential tools to constrain a specific Higgs-curvature connection. The most promising seems to be a reanalysis of the redshifted signals during GRB events, and analysis of recent data taken with gamma-ray observatories could be targeted looking for this peculiar effect. This suggests the need for a comprehensive reanalysis of gamma-ray bursts in which transient features appear in the energy spectra. There is tension between the various observational parameters involved, as one simultaneously makes three demands: high time resolution to avoid washing out the transient in case of a sampling time too large, high energy resolution to identify with enough precision the location of the lines, and large statistics to avoid the signal being immersed in the background. This also adds motivations to the development of satellite detectors in the $1 \mathrm{keV}-10$ $\mathrm{MeV}$ range with high spectral and temporal resolutions, and large gamma spectrometers on balloons [54-57] in which the shorter observation time could be offset by the larger fiducial detection volume, or the 100-day observation time planned for the Ultra Long Duration Balloon program ([58-60], See for instance [61]). An alternative method could be the simultaneous observation of $e^{+} e^{-}$and $p \bar{p}$ annihilations, and we 
have shown that bounds are of similar order of magnitude if state of art instrumental resolution can be achieved.

We also mention the feasibility of observations of cyclotron lines of electrons and protons or ions in the same region of magnetic fields of neutron stars. So far there have been observations of both lines but in different neutron stars; see [62] for the evidence of a line feature in a soft gamma repeater interpreted as a proton cyclotron resonance, [63] for a feature from a magnetar also interpreted as a proton cyclotron feature, and [64] for a band of electron cyclotron lines from an isolated neutron star. The mismatch of the two cyclotron frequencies by the proton-to-electron mass ratio makes their simultaneous observation on the same neutron star quite difficult. This mismatch is smaller for electron and proton spinflip resonances, however, qualitative estimates show that their absorption signal is suppressed, with respect to the one due to the cyclotron resonance, by a factor approximately equal to the fine structure constant, ruling out its observability with the current data [65-67].

The work discussed so far on Higgs shifts based on a Kretschmann coupling can also be extended in the analysis of bounds to Higgs-curvature couplings with the Ricci scalar for models beyond general relativity. ${ }^{2}$ The JebsenBirkhoff theorem, i.e. the fact that the Schwarzschild solution is the unique spherically symmetric vacuum solution, does not hold in metric $f(R)$ gravity, and $R \neq 0$ even if $T=0$ (see [69] for a dedicated study of possible counterexamples). This means that a putative observed Higgs shift will be differently interpreted in various approaches, as due to the Higgs-Kretschmann coupling in ordinary general relativity, of as a Ricci-Higgs coupling in higher dimensional theories [70,71], $f(R)$ theories [72,73], or in Hořava gravity [74-76]. Further observables will therefore be necessary to disentangle the various theoretical scenarios.

Acknowledgments We are grateful to J.M. Lattimer for useful correspondence, and to S. Lenzi , R. M. Millan, and R. Turolla for helpful discussions.

Open Access This article is distributed under the terms of the Creative Commons Attribution 4.0 International License (http://creativecomm ons.org/licenses/by/4.0/), which permits unrestricted use, distribution, and reproduction in any medium, provided you give appropriate credit to the original author(s) and the source, provide a link to the Creative Commons license, and indicate if changes were made. Funded by SCOAP ${ }^{3}$.

\section{References}

\section{F. Bezrukov, M. Shaposhnikov, Phys. Lett. B 659, 703 (2008)}

\footnotetext{
2 We need to point out that, according to the discussion presented in [68], the presence of nonminimal couplings between spacetime curvature and any quantum field gives rise to violations of the equivalence principle putting the models outside the realm of general relativity.
}

2. F. Bezrukov, A. Magnin, M. Shaposhnikov, Phys. Lett. B 675, 88 (2009)

3. G. Aad et al., ATLAS Collaboration, Phys. Lett. B 710, 49 (2012)

4. S. Chatrachyan et al., CMS Collaboration, Phys. Lett. B 710, 26 (2012)

5. G. Degrassi, S. Di Vita, J. Elias-Miró J. R. Espinosa, G. F. Giudice, G. Isidori, A. Strumia, J. High Energy Phys. 08, 098 (2012)

6. V. Branchina, E. Messina, Phys. Rev. Lett. 111, 241801 (2013)

7. D. Buttazzo, G. Degrassi, P.P. Giardino, G.F. Giudice, F. Sala, A. Salvio, A. Strumia, J. High Energy Phys. 12, 089 (2013)

8. M. Holthausen, K.S. Lim, M. Lindner, J. High Energy Phys. 12, 037 (2012)

9. F. Wang, Nucl. Phys. B 884, 193 (2014)

10. D.A. Demir, Phys. Lett. B 733, 237 (2014)

11. M. Torabian, arXiv: 1410.1744

12. P.A.R. Ade et al., BICEP Collaboration, Phys. Rev. Lett. 112, 241101 (2014)

13. R. Adam et al., Planck Collaboration, arXiv: 1409.5738

14. J. Espinosa, G. Giudice, A. Riotto, JCAP 0805, 002 (2008)

15. M. Fairbairn, R. Hogan, Phys. Rev. Lett. 112, 201801 (2014)

16. R. Onofrio, G.A. Wegner, Astrophys. J. 791, 125 (2014)

17. B.J. Teegarden, T.L. Cline, Astrophys. J. 236, L67 (1980)

18. B.J. Teegarden, T.L. Cline, Astrophys. Space Sci. 75, 181 (1981)

19. E.P. Mazets, S.V. Golenetskii, R.L. Aptekar, Yu. A. Guryan, V. N. Ilinskii. Nature 290, 378 (1981)

20. S.V. Golenetskii, E.P. Mazets, R.L. Aptekar, YuA Guryan, V.N. Ilyinskii, Astrophys. Space Sci. 124, 243 (1986)

21. B. Kozlovsky, R.J. Murphy, R. Ramaty, Astrophys. J. 141, 523 (2002)

22. W.M. Johnson III, F.R. Harnden, R.C. Haymes, Astroph. J. Lett. 172, L1 (1972)

23. R. Ramaty, G. Borner, J.M. Cohen, Astrophys. J. 181, 891 (1973)

24. D.M. Palmer et al., Astrophys. J. 433, L77 (1984)

25. K. Hurley, Astrophys. J. Suppl. Ser. 90, 857 (1994)

26. B.J. Teegarden, Astrophys. Space Sci. 231, 137 (1995)

27. E.E. Fenimore, R.W. Klebesadel, J.G. Laros, Adv. Space Res. 3, 207 (1983)

28. B.J. Teegarden, in High Energy Transients in Astrophysics, AIP Conf. Proc. No. 115, ed. by S.E. Woosley (American Inst. Phys., New York, 1984), pp. 352-356

29. T.J. Loredo, R.I. Epstein, Astrophys. J. 336, 896 (1989)

30. A.A. Zdziarski, in Proceedings of 13th Texas Symposium Relativistic Astrophysics, ed. by M. Ulmer (World Scientific, Singapore, 1987), pp. 553-562

31. D.Q. Lamb, in Nuclear Spectroscopy of Astrophysical Sources, ed. by N. Gehrels, G.H. Share (America Inst. Phys., New York, 1988), pp. $265-284$

32. G.J. Fishman, R.D. Clayton, Astrophys. J. 178, 337 (1972)

33. R. Ramaty, J.M. McKinley, F.C. Jones, Astrophys. J. 256, 238 (1982)

34. F.A. Aharonyan, A.M. Atoyan, R.A. Sunyaev, The annihilation spectrum of relativistic electron-positron plasma. Yerevan Phys. Inst. 432(39), 80 (1980)

35. A.A. Zdziarski, Acta Astron. 30, 371 (1980)

36. R. Ramaty, P. Mészáros, Astrophys. J. 250, 384 (1981)

37. R. Svensson, Astrophys. J. 258, 335 (1982)

38. F.A. Aharonyan, A.M. Atoyan, R.A. Sunyaev, Astrophys. Space Sci. 93, 279 (1983)

39. E.P. Mazets et al., Astrophys. Space Sci. 80, 3 (1981)

40. E.P. Mazets et al., Astrophys. Space Sci. 80, 85 (1981)

41. E.P. Mazets et al., Astrophys. Space Sci. 80, 119 (1981)

42. N.K. Glendenning, Phys. Rev. D 46, 1274 (1992)

43. J.M. Lattimer, M. Prakash, D. Masak, A. Yahil, Astrophys. J. 355, $241(1990)$

44. A.W. Steiner, J.M. Lattimer, E.F. Brown, Astrophys. J. 722, 22 (2010) 
45. J.M. Lattimer, A.W. Steiner, Astrophys. J. 784, 123 (2014)

46. J.M. Lattimer, A.W. Steiner, Eur. Phys. J. A 50, 40 (2014)

47. R. Onofrio, Phys. Rev. D 82, 065008 (2010)

48. R. Onofrio, Eur. Phys. J. C 72, 2006 (2012)

49. E. Caurier, G. Martinez-Pinedo, F. Nowacki, A. Poves, A.P. Zuker, Rev. Mod. Phys. 77, 427 (2005)

50. N. Arkani-Hamed, S. Dimopoulos, G. Dvali, Phys. Lett. B 429, 263 (1998)

51. N. Arkani-Hamed, S. Dimopoulos, G. Dvali, Phys. Rev. D 59, 086004 (1999)

52. G. Backenstoss et al., Nucl. Phys. B 228, 424 (1983)

53. E. Churazov, R. Sunyaev, S. Sazonov, M. Revnivtsev, D. Varshalovich, Mon. Not. R. Astron. Soc. 357, 1377 (2005)

54. G.J. Fishman, R.W. Austin, Nucl. Instr. Meth. 140, 193 (1976)

55. G.J. Fishman, J.W. Watts, Astrophys. J. 212, 211 (1977)

56. G.J. Fishman, C.A. Meegan, J.W. Watts, J.H. Derrickson, Astrophys. J. 223, L13 (1978)

57. C.A. Meegan, G.J. Fishman, R.C. Haymes, Astrophys. J. 234, L123 (1979)

58. H.M. Cathey, Adv. Space Res. 33, 1633 (2004)

59. H. Fuke et al., Adv. Space Res. 53, 1432 (2014)

60. T. Aramaki et al., Astropart. Phys. 59, 12 (2014)

61. Columbia Scientific Balloon Facility (2015). http://www.csbf.nasa. gov/balloons.html
62. A.I. Ibrahim et al., Astrophys. J. 574, L51 (2002)

63. A. Tiengo et al., Nature 500, 312 (2013)

64. G.F. Bignami, P.A. Caraveo, A. de Luca, D. Meneghetti, Nature 423, 725 (2003)

65. D.B. Melrose, V.V.Zheleznyakov, Astron. Astrophys. 95, 86 (1981)

66. C. Thompson, in The Neutron Star-Black Hole Connection, Proceedings of the NATO Advanced Study Institute, ed. by C. Konveliotou, J. Ventura, E. Van den Heuvel. NATO Science Series C: Mathematical and Physical Sciences, vol. 567 (Kluwer, Dordrecht, 2001), p. 369

67. S. Zane, R. Turolla, L. Stella, A. Treves, Astrophys. J. 560, 384 (2001)

68. E. Okon, C. Callender, Eur. J. Philos. Sci. 1, 133 (2011)

69. H. Goenner, P. Havas, J. Math. Phys. 42, 1837 (2001)

70. I.K. Wehus, F. Ravndal, Int. J. Mod. Phys. A 19, 4671 (2004)

71. I.K. Wehus, F. Ravndal, J. Phys.: Conf. Ser. 66, 012024 (2007)

72. V. Faraoni, Phys. Rev. D 81, 044002 (2010)

73. A.M. Nzioki, R. Goswami, P.K.S. Dunsby, Phys. Rev. D 89, 064050 (2014)

74. P. Hořava, Phys. Rev. Lett. 102, 161301 (2009)

75. P. Hořava, Phys. Rev. D 79, 084008 (2009)

76. D.L. López-Nacir, F.D. Mazzitelli, L.G. Trombetta, Phys. Rev. D 85, 024051 (2012) 